This PDF is a simplified version of the original article published in Internet Archaeology. Enlarged images which support this publication can be found in the original version online. All links also go to the online version.

Please cite this as: Calandra. E., Boi, V., Falcone, A., Acconcia, V., Di Giorgio, S., Massara, F. and Ronzino, P. 2021 Policy and Practice for Digital Archaeological Archiving in Italy, Internet

Archaeology 58. https://doi.org/10.11141/ia.58.27

\title{
Policy and Practice for Digital Archaeological Archiving in Italy
}

Elena Calandra, Valeria Boi, Annalisa Falcone, Valeria Acconcia, Sara Di Giorgio, Flavia Massara and Paola Ronzino

This article highlights how the Italian Central Institute for Archaeology (ICA) is developing the National Geoportal for Archaeology (GNA), based on the ARIADNEplus infrastructure and its policy framework. Thanks to the GNA project, it will be possible to search and learn about archaeological documentation managed by Superintendencies and Universities holding a significant amount of archaeological data, much of which is either completely or partially unpublished.

\section{Introduction}

In recent decades, the so-called 'digital revolution' has profoundly transformed the ways in which archaeological data are published, consulted and reused. Digital technologies facilitate access to data and documents, without the limitations and inconveniences caused by the need to directly access paper archives. However, these advantages run the risk of remaining purely theoretical as this paradigm shift seems to have only partially affected the daily activities of archaeologists in the field, both in terms of the quantity and quality of the data actually available for open access on the internet. Yet an increase in the availability of these resources could stimulate and make academic exchanges and discussions in international spheres more profitable, and, last but not least, promote better professional and research conditions.

The low cost and ease of access to online digital data could enable an actual 'democratisation of knowledge'. This could reduce the existence of archives that are 
normally accessible only to a limited number or to certain categories of users, thus leading to an exponential increase in the information potential of such data and documents in terms of the ability to create and share such knowledge.

The health emergency caused by the spread of COVID-19 has further highlighted how the ability to access data and documents in digital formats (preferably open access) has, when access to paper archives becomes impossible, proven to be indispensable for the continuation of research and safeguarding activities under particular conditions. Since March 2020 and through the following months of lockdown, the work of so-called 'intellectual professions' has been carried out entirely remotely, and the enormous resource represented by the functionalities of online platforms was quite evident. The safeguarding activities carried out by the Ministry of Culture $(\mathrm{MiC})$ personnel were guaranteed only by the availability of online digital tools, both for purely administrative functions (documentation register, correspondence management) and on the scientific side (consultation of documents, issuing technical advice, continuation of research planning activities, and collaborations with other Institutes). Thus, the offices already equipped with digital tools were able to ensure a greater continuity compared to those offices that did not have any, or only partially had them. This experience has therefore highlighted the extreme variability of situations at the national level, where there are offices that have had consolidated repositories and digital platforms for years, and other offices in which it is difficult to find even digitised indexes of the documentation kept at the paper archives.

The management of the scientific documentation delivered to the MiC at the conclusion of field investigations also varies greatly. In this context, the request to produce georeferenced plans of excavations and finds in geospatial formats that constitute the basis for a national archaeological map that can be easily updated and implemented over time, is still not widespread enough. In addition, standardised criteria for data accessibility have not yet been adopted. Consequently, information concerning interventions for the protection of archaeological heritage are only rarely published on online platforms. The extreme variability of the solutions adopted, ranging from open access to topographical data and scientific documentation (the so-called grey literature), up to the availability of only a short description and representation of data, can also be observed. Finally, repositories and databases reserved only for internal use are still the most widespread. This article will attempt to briefly outline the state of the art in the field of archaeological digital archives in Italy. It will also explain the approach adopted by the project for the creation of the National Geoportal for Archaeology.

\section{Legal Framework and Administrative Context}

Before delving into an analysis of the problem, it is appropriate to briefly present the various actors involved in the production and management of archaeological data in Italy. The execution of archaeological excavations is the responsibility of the State, represented by the Ministry of Culture (MiC), which is fundamentally tasked with the protection, use, and enhancement of cultural heritage. The MiC carries out its 
activities through the Directorate General for Archaeology, Fine Arts and Landscape (DG ABAP). In particular, the protection of archaeological heritage is undertaken through its Service II, which plays a coordinating role in relation to the local offices: the Superintendencies. The latter take on the scientific direction of archaeological research, both in the context of excavations and other planned interventions, and in the case of preventive and emergency archaeology and archaeological supervision during land and urban transformation (renewal) works. In all cases, scientific direction refers to the coordinating role of the Superintendencies during the excavation, which is only waived in the case of the so-called 'excavations in concession', in which a University or other research body is allowed to take on the scientific direction of the research, usually entrusted to the Ministry of Culture's local offices.

As far as digital archiving is concerned, the Central Institute for Archaeology (ICA), established in 2016 (Ministerial Decree no. 245 of 13 May 2016), has among its institutional tasks:

- the definition and application of guidelines, standards and coordination measures for the development of studies and research in the field of archaeology;

- the assessment of archaeological data archives in Italy, as well as their publication in open data format;

- proceeding with the systematic recovery of previous documentation and the definition of the terms of the rights of publication, as well as the development of databases and thematic maps for the purpose of creating a unified map of the archaeological potential on a national scale (Ministerial Decree no. 169 of 7 April 2017, n. 169).

It is therefore a role strictly connected with the protection tasks carried out by Service II of DG ABAP and by the local Superintendencies, with the aim of promoting the standardisation of workflows and documentation and fostering their open access publication.

As already mentioned, although preventive archaeology and archaeological supervision during land and urban transformation are carried out under the scientific direction of the $\mathrm{MiC}$ and its local Superintendencies, the fieldwork is rarely carried out by its own staff and usually this is the most frequent context in which new archaeological finds emerge in Italy. In fact, excavations are normally carried out by private companies or freelancers with funds allocated by the clients of the works (Legislative Decree 50/2016, 'Code of Public Contracts'). Planned archaeological research activities, on the other hand, are mainly carried out by universities and other research bodies, from both within and outside Italy, which can carry out field activities as part of a concession issued by the $\mathrm{MiC}$, which sets out the scientific direction of the research (Legislative Decree 42/2004, art. 88 and 89; in particular, the DG-ABAP Service II is in charge of the 'concession to public or private entities for the execution of archaeological research or works aimed at the discovery of cultural assets' (D.P.C.M. 2 December 2019, n. 169, art. 16, paragraph 2, lett. E)).

Tthe collection and management of archaeological data in Italy is therefore entrusted to various organisationsinstitutes operating within the Ministry of Culture but also to other actors, institutional and otherwise, involved in various capacities during the diverse phases of research. 


\section{The Design Process of the National Geoportal for Archaeology (GNA)}

The National Geoportal for Archaeology (GNA) project is part of a broader project for the standardisation of scientific documentation produced within the scope of all archaeological research carried out in Italy. Its design was developed through a long phase of technical and methodological reflections concerning the creation of a single national infrastructure for the collection and publication of archaeological data. The most important aspects of the activity happened during two inter-ministerial commissions, consisting of representatives of the Ministry of University and Scientific Research and of the Ministry of Culture, operating between 2007 and 2010. Some of the most important open access archaeological databases currently existing in Italy can be considered as the outcome of this period of theoretical elaboration and experimentation: the SITAR in Rome, the RAPTOR project, the MAPPA project in Pisa, and the web platforms with restricted access developed by Universities and Research Institutes (SITAN). Over the years, however, the possibility of achieving real interoperability between the various databases has conflicted with the difficulty of surveying and standardising the enormous amount of pre-existing data stored in various archives. Even more critical was normalising new data and attaining the definition of a minimum standard set in a native digital format to allow continuous updates of the database, with costs and resources (both technical and human) that can be sustained by the institutions involved.

Starting from these initial experiences, the GNA was born with the primary aim of collecting reports on the interventions carried out under the scientific direction of the MiC (preventive archaeology, archaeological supervision during the excavation work, fortuitous finds and planned excavations), yet it is also structured to accommodate the results of studies conducted by universities and other research bodies, with the view of setting up a single national access point to the base data of archaeological research. The main objective of the GNA is to make the protection of archaeological heritage simpler and more efficient by improving the tools used to access and visualise the information on a topographic platform.

Implementation of the project began when an agreement was signed between ICA, the Central Institute for the Single Catalogue of Italian Libraries and Bibliographic Information (ICCU) and PIN - VastLab - Polo Universitario di Prato on 20 October 2017. It was further strengthened in August 2018 by the European Commission's approval of the ARIADNEplus project, to which ICA joined as an affiliated entity of ICCU for the MiC. Within the ARIADNEplus project, the Geoportal represents the Italian pilot experiment for the creation of the geographic database that enables searching for information via a map-based interface. The implementation of this pilot scheme was entrusted to the NemiS laboratory of CNR-ISTI in Pisa, which is experimenting with data management functions via geographical maps and adding features for cartography-based research and geospatial data management. 


\section{Data Model and Information Categories}

In 2018, when collection of data from the Superintendencies to be published on the Geoportal began, the first dataset selected for testing was represented by preventive archaeology (preventive archaeology procedures, carried out according to c. 4 of art. 28 of Legislative Decree 42/2004 and regulated by art. 25 of Legislative Decree $50 / 2016)$. This category of information constitutes one of the preferred ways in which a huge mass of archaeological data about a territory, quite often unpublished, has been collected in Italy over the past 15 years and in most cases originating in archival research and territorial surveys. Governed by the Malta Convention, which affirms it is up to the institutional body about to irreversibly modify an area for the construction of public infrastructure to bear the costs of the scientific investigation, preventive archaeology also represents a particular case in terms of the 'obligation' to disseminate the data collected. In Italy, the Malta convention was upheld with Law $57 / 2014$. The first rule on the management of preventive archaeology interventions, however, precedes the ratification of the Convention and is represented by Law 109/2005. At national level, the Circular 1/2016 of the former DG-Archaeology established that '...the documentation of direct and indirect investigations...must be sent to the Archaeological Superintendency responsible for the area on paper, as well as via digital formats, according to the formats defined by MiBACT (now MiC). The results of such surveys will be published immediately in a digital archive and made available through a freely accessible web platform'. Based on the Ministry's guidelines, ICA started (in collaboration with the Central Institute for the Catalogue and Documentation (ICCD) (Mancinelli 2018) and Service II of DG-ABAP) to identify such data, starting from the archives of the Superintendencies of three 'pilot regions': Piedmont, Tuscany and Puglia.

The definition of the data model was entrusted to a joint ICA-ICCD-Service II DGABAP working group. The standard adopted is the MODI (Information Module) of ICCD, created to allow the expeditious inventory of data in a pre-cataloguing phase, which requires a greater level of in-depth analysis and study. With a structure that can be adapted to any cultural property, whether movable or immovable, MODI has been 'customised' to make it more suitable for users in terms of speed and ease of use by inserting in the original form some additional fields - either borrowed from ICCD's standard Site module (SI 4.00) or created from scratch when necessary to improve the description of specific aspects of preventive archaeology procedures. The need for standardising and normalising information that enables the optimisation of the search features within the GNA database has orientated the developers towards the definition of controlled vocabularies that guide the choice of cataloguers, thus avoiding the multiplication of terms whose future management would be very complex, both in the data-entry phase and in the retrieval of information.

Two distinct models to distinguish between administrative and scientific data were developed using MODI as a starting point: the MOPR - Project Module - collects information about the public works from which the procedure originated; and the MOSI - archaeological Site Module - stores the data about the individual 
sites/archaeological areas identified in the area affected by the project. Thus a oneto-many relationship is created between the project and all the archaeological information collected in the area affected by the works.

In structuring the two modules, it was decided that some essential information was compulsory:

1. the type of work and its commissioning body;

2. the type of research carried out in the area and sources used for collecting information, chronology, extension, topographic;

3. the altimetric data about the archaeological record;

4. the distance from the modern infrastructure that is going to be built;

5. the assessment of the impact of the infrastructure itself on the archaeological record/landscape.

Georeferencing of both the project area and the archaeological sites is fundamental in view of a future integration of the data collected with other topographical information about Italian cultural heritage held by the Ministry and of their future publication within the Geoportal. Information regarding all those who collaborated in the collection, analysis and processing of the data is also recorded, i.e. the officer responsible for the preventive archaeology procedure, the archaeological professionals in charge of drafting the archaeological report, and the compilers of the form.

Additionally, upon request by the local archives, the data model consisting of the MOPR and MOSI modules was also adopted for the census of other data stored. This expanded the data-entry to include other cultural heritage protection categories (emergency excavations, archaeological supervision during the excavation works) and to include the data directly collected by the offices themselves or in collaboration with universities, research institutes and individual scholars for research purposes. Regardless of the acquisition methods and the context in which they are studied or excavated, archaeological data can be archived and searched according to a data structure that has been adopted at the international level, from two points of view: the event/circumstance of data collection (event) and the data itself (archaeological record). Between the event and the archaeological record, there is a many-tomany relationship in the database, since an archaeological site/monument can be investigated over time from $n$ events (excavations, surveys, other studies), with different methods and different interpretative outcomes, while on the other hand during a single large-scale excavation, especially in development-led archaeology, many archaeological records (sites, monuments) can be created. The data model adopted by the Geoportal makes it possible to update the information relating to both levels by implementing the system with new data and 'versioning' the old ones.

The publication of the data on the Geoportal also involves the data collected in the context of the excavations carried out by universities and research bodies that have been granted a concession (Figure 1). In this case, the Ministry of Culture transfers the scientific direction of the research to the research body that carries out the archaeological investigations, so that the publication of the results answers its primary purpose. This includes guaranteeing the dissemination of the baseline information about the research conducted on national soil, while respecting the right/obligation of the research team to delve deeper into the research and publish 
the results in the most appropriate context. For this purpose, Service II DG ABAP and the ICA proceeded to renew the process of standardisation and digitisation of the entire documentation related to the procedure, both on the administrative and scientific fronts (http://www.ic archeo.beniculturali.it/it/231/circolari-e-modulistica).

During the work of defining new standards for archaeological documentation, with the aim of publishing it on the GNA, files are geo-referenced in the WGS84 reference system, as well as the complete metadata containing an exhaustive description of the dataset (content, methods of acquisition and access, authors and managers, funding). This will make it possible to connect the digital resources to each other and to carry out accurate searches on the basis of shared standards and vocabularies, thus increasing the level of searchability, accessibility, interoperability and the reuse of data in line with the FAIR data principles (Wilkinson et al. 2016). Our efforts, therefore, focused on identifying the minimum set of information that could be made usable in an open format so as to represent a basic research project. By uploading it to the GNA, it will return a snapshot of archaeological research in Italy that can be useful both for protection of archaeological resources and for the dissemination and comparison of data between the various actors undertaking the research.

For archaeological research carried out under a regime of ministerial concession, the updating of the procedures described thus far (introduced in the operational practices of the Ministry with Circular no. 30/2019 of the General Directorate of Archaeology, Fine Arts and Landscape, and merged into the most recent and currently in force circular 14/2021 of the same Directorate) also introduces new guidelines for publishing the research. Assuming that the possibility of processing and disseminating research data is part of the concession (pursuant to Article 88 of Legislative Decree 42/2004), the MiC is tasked with supervising the forms of dissemination. In this way, the cultural heritage protection is granted, and at the same time the concessionaires can give visibility to the results of the surveys through open access publications, scientific editions, university lectures, conferences, interviews and broadcasts on radio and television, social media, blogs and websites. To facilitate the dissemination of research data, and as an incentive to close the gap between the investigations and dissemination of the results, the same circular letter also gives the excavation director the opportunity to publish the annual report in the form of an article in the periodical published by the MiC itself; the New Online Archaeology Bulletin. 


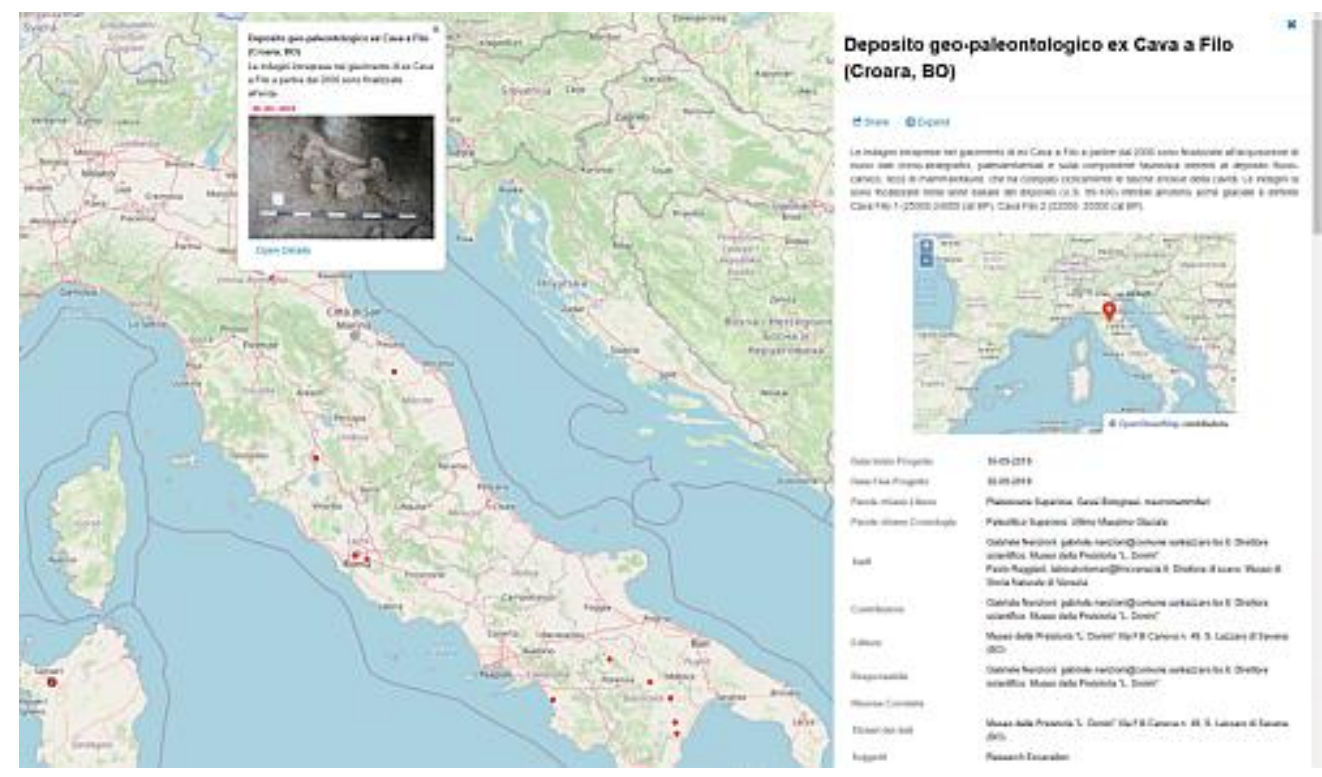

Figure 1: The Geoportal and a sample record of archaeological excavations

\section{Accessibility}

A final topic of primary importance is the level of data openness. The Geoportal is structured as a tool that Ministry personnel can use to enter, verify and consult data, while external users will be able to enter their own data and consult the existing information. The portal will therefore have different levels of access and will require the detailed definition of various aspects, both for updating and consulting the data.

The minimum set of data for each research intervention or archaeological site/monument will be accessible to all users and will include the georeferencing, description, interpretation, chronology, author/s and responsible agencies. It will be published for all data collected as part of the ministerial activity but also for academic projects published by the partners. Accredited users will be able to access the link to the attached documentation at a detailed cartographic level, which is expected to show not only a point but the polygons showing the real extent of the investigations carried out and/or the structures identified (unless there are specific requirements for the protection of the archaeological sites). The exact position of archaeological sites will not be available on the open access portal in particular cases e.g. sites located in remote/rural areas, necropolises, and underwater deposits. In such cases, scaledependent visualizations will be adopted, which will make only the approximate position of the site publicly available, to protect contexts and to prevent theft or illegal excavation.

\section{Conclusions}

The data model adopted by the National Geoportal for Archaeology will be used for all archaeological research data collected in Italy, starting with those directed by the $\mathrm{MiC}$ and from excavations under ministerial concession. The GNA comprises the first national digital database of archaeological research in Italy, and its main aims, useful both for protection and research, are as follows: 
- creation of a minimum knowledge base about archaeological heritage, which can be easily shared and updated;

- $\quad$ support for heritage protection activities regarding data accessibility and document storage. It represents the first solution addressing the obsolescence of digital formats and hardware;

- dissemination of description/representation standards aligned with the National Catalogue System (ICCD) and with international standards (through the ARIADNEplus project) (Felicetti et al. 2013);

- definition of minimum levels of access to data for various categories of external users.

The collaboration with the ARIADNEplus consortium is adding an international framework to the GNA project, making it possible to analyse and adopt standards and guidelines that have already been successfully adopted in other countries and, on a scientific level, building the promise of real interoperability between archaeological databases created in different countries (Acconcia et al. 2019).

\section{Bibliography}

Acconcia, V., Boi, V., Di Giorgio, S., Falcone, A. and Ronzino, P. 2019 'The Italian Geoportal for archaeology: a new tool for data integration and reuse' in J.D. Richards and F. Niccolucci (eds) The ARIADNE Impact. Budapest: Archaeolingua. 8392. https://doi.org/10.5281/zenodo.3476712

Felicetti, A., Scarselli, T., Mancinelli, M.L. and Niccolucci, F. 2013 'Mapping ICCD archaeological data to CIDOC-CRM: the RA Schema' in V. Alexiev, V. Ivanov and M. Grinberg CRMEX 2013. Proceedings of the Workshop Practical Experiences with CIDOC CRM and its Extensions co-located with the 17th International Conference on Theory and Practice of Digital Libraries, Valetta, Malta, September 26, 2013, 1122. http://ceur-ws.org/Vol-1117/ (PDF)

Mancinelli, M.L. 2018 'Gli standard catalografici dell'Istituto Centrale per il Catalogo e la Documentazione' in R. Tucci Le voci, le opere e le cose. La catalogazione dei beniculturali demoetnoantropologici. http://www.iccd.beniculturali.it/getFile.php?id=6457

Wilkinson, M., Dumontier, M., Aalbersberg, I. et al. 2016 The FAIR Guiding Principles for scientific data management and stewardship. Scientific Data 3(1), 160018. https://doi.org/10.1038/sdata.2016.18 